\title{
Carcinoma mamário pouco diferenciado em macaco-prego, Cebus sp. (Cebidae) ${ }^{1}$
}

\author{
Liane Ziliotto ${ }^{2 *}$, Marcos V. Tranquilim², Ewerton L. Lima², Paulo C.D. Vecchia², \\ Ricardo C. Lehmkuhl ${ }^{2}$ e Adriano O.T. Carrasco ${ }^{2}$
}

\begin{abstract}
Ziliotto L., Tranquilim M.V., Lima E.L., Vecchia P.C.D., Lehmkuhl R.C. \& Carrasco A.O.T. 2013. [Mammary high-grade carcinoma in a monkey, Cebus sp. (Cebidae).] Carcinoma mamário pouco diferenciado em macaco-prego, Cebus sp. (Cebidae). Pesquisa Veterinária Brasileira 33(1):99-104. Departamento de Medicina Veterinária, Universidade Estadual do Centro-Oeste, Rua Simeão Camargo Varella de Sá 3, Guarapuava, PR 85040080, Brazil. E-mail: lianeziliotto@yahoo.com.br

Mammary tumors in non-human primates are rare. The aim of this paper is to report the occurrence and treatment outcomes of a female Cebus sp. (fam. Cebidae) with high-grade mammary carcinoma received at the Serviço de Atendimento de Animais Selvagens (SAAS), Universidade Estadual do Centro-Oeste. A right mammary gland nodule was identified and at radiographic examination a point of radiopacity due to gun projectile entrapped within the mammary mass was seen. After the patient stabilization, the mass was excised and submitted to histopathological examination. The diagnosis was of high-grade carcinoma, compatible with adenosquamous carcinoma. The monkey was transported to Wild Animals Treat and Receiving Center (CETAS) and introduced into a colony. More than 20 months after surgery no evidence of relapse was seen and the patient is living with the group. That suggests that the adopted therapy was effective, achieving quality of life and increased survival.
\end{abstract}

INDEX TERMS: Oncology, mammary tumor, carcinoma, wild animals, primates, capuchin monkey, $\mathrm{Ce}$ bus sp., Cebidae.

RESUMO.- As neoplasias mamárias são raras em primatas não humanos, enquanto que nas mulheres apresentam alta incidência. 0 objetivo deste trabalho foi relatar a ocorrência e os resultados do tratamento de um Cebus sp. (fam. Cebidae) fêmea, com carcinoma mamário pouco diferenciado encaminhado ao Serviço de Atendimento de Animais Selvagens da Universidade Estadual do Centro-Oeste. À inspeção o animal apresentava aumento de volume em região mamária direita e ao exame radiográfico foram observados três pontos de radiopacidade, característicos de projétil balístico de arma de pressão, com um deles alojado ao centro da massa de tecido mamário Após estabilização, a paciente foi submetida à exérese cirúrgica de aumento de volume e o material foi encaminhado para análise histopatológica. 0 diagnóstico obtido foi de carcinoma de alto grau, compa-

\footnotetext{
${ }^{1}$ Recebido em 27 de junho de 2012.

Aceito para publicação em 5 de setembro de 2012.

${ }^{2}$ Departamento de Medicina Veterinária, Universidade Estadual do Centro-Oeste, Rua Simeão Camargo Varella de Sá 3, Guarapuava, PR 85040080, Brasil. E-mail: lianeziliotto@yahoo.com.br
}

tível com carcinoma adenoescamoso. Após a retirada dos pontos a paciente foi encaminhada ao convívio de outros animais. Mais de 20 meses após a terapia cirúrgica não há sinais de recidiva. A paciente alimenta-se bem, convive normalmente com o grupo, sugerindo que a terapia adotada foi eficiente até o momento em alcançar qualidade de vida e aumento de sobrevida do animal.

TERMOS DE INDEXAÇÃO: Oncologia, tumor mamário, carcinoma, animais selvagens, primatas, macaco-prego, Cebus sp., Cebidae.

\section{INTRODUÇÃO}

Em seres humanos a incidência mundial de câncer de mama em 2008 foi de um milhão duzentos e noventa mil casos de acordo com a Organização Mundial de Saúde (OMS). Segundo as estimativas do Inca/Ministério da Saúde, no Brasil a incidência de neoplasias mamárias em mulheres para o ano de 2012 será de mais de 52 mil novos casos, tornando este tipo de tumor a neoplasia mais frequente em mulheres (Brasil 2012). Contudo, em primatas não humanos, as neoplasias mamárias não são tão fre- 
quentes e são predominantemente malignas, com maior incidência das de origem epitelial. Os relatos de tumores mamários nestes indivíduos são raros, e mais frequentes em primatas do velho mundo (Catarrhini), como fibroadenocarcinoma e carcinomas em macaco Rhesus e babuínos (Lewis \& Colgin 2005). Nos primatas do novo mundo (Platyrrhini) foi encontrado o relato de apenas um caso de adenocarcinoma mamário em macho de macaco-de-cheiro (Saimiri sciureus) (Waggie et al. 2000). 0 "macaco-prego" ou "mico preto" é um primata do novo mundo que pertence à família Cebidae que é dividida em três subfamílias: Cebinae, Saimirinae e Callitrichinae. 0 gênero Cebus, ao qual o "macaco-prego" pertence, é composto por seis espécies ocorrentes no Brasil, que vivem em todos os tipos de florestas neotropicais, principalmente na Floresta Amazônica (Bicca-Marques et al. 2006). São mamíferos placentários, com cauda semi-preênsil, arborícolas, com um par de mamas localizadas no tórax e raramente o número de filhotes é maior que dois (Verona \& Pissinati 2006). Nas fêmeas a genitália apresenta-se bem desenvolvida e pendular e pode-se confundir com estrutura peniana (Bicca-Marques et al. 2006, Verona \& Pissinati 2006). Podem chegar a pe- sar de dois a quatro quilogramas de peso, e na natureza se alimentam de sementes e frutos de casca dura ou mole, porém, em cativeiro são onívoros (Rocha et al. 1998). Estão entre os mamíferos mais facilmente encontrados em pequenas extensões florestais do estado do Paraná, ocupando desde grandes remanescentes florestais até matas contínuas (Passos et al. 2006). Rocha (2000) acredita que a espécie pode sobreviver em pequenas áreas florestais desde que tenha acesso a pomares e plantações adjacentes, como plantios de milho, cana-de-açúcar e mandioca, de onde os animais obtêm um importante suplemento alimentar. Em algumas regiões do estado, tem sido considerada como praga por produtores agrícolas ao invadir e atacar plantações e pomares, até mesmo monoculturas de Pinus sp. (Koehler \& Firkowski 1996, Rocha 2000). Não foram encontradas referências de neoplasias mamárias em indivíduos desta espécie e, desta forma, objetiva-se com este trabalho relatar a ocorrência e os resultados do tratamento de um Cebus sp. fêmea, com carcinoma mamário pouco diferenciado atendida pelo Serviço de Atendimento de Animais Selvagens da Universidade Estadual do Centro-Oeste.

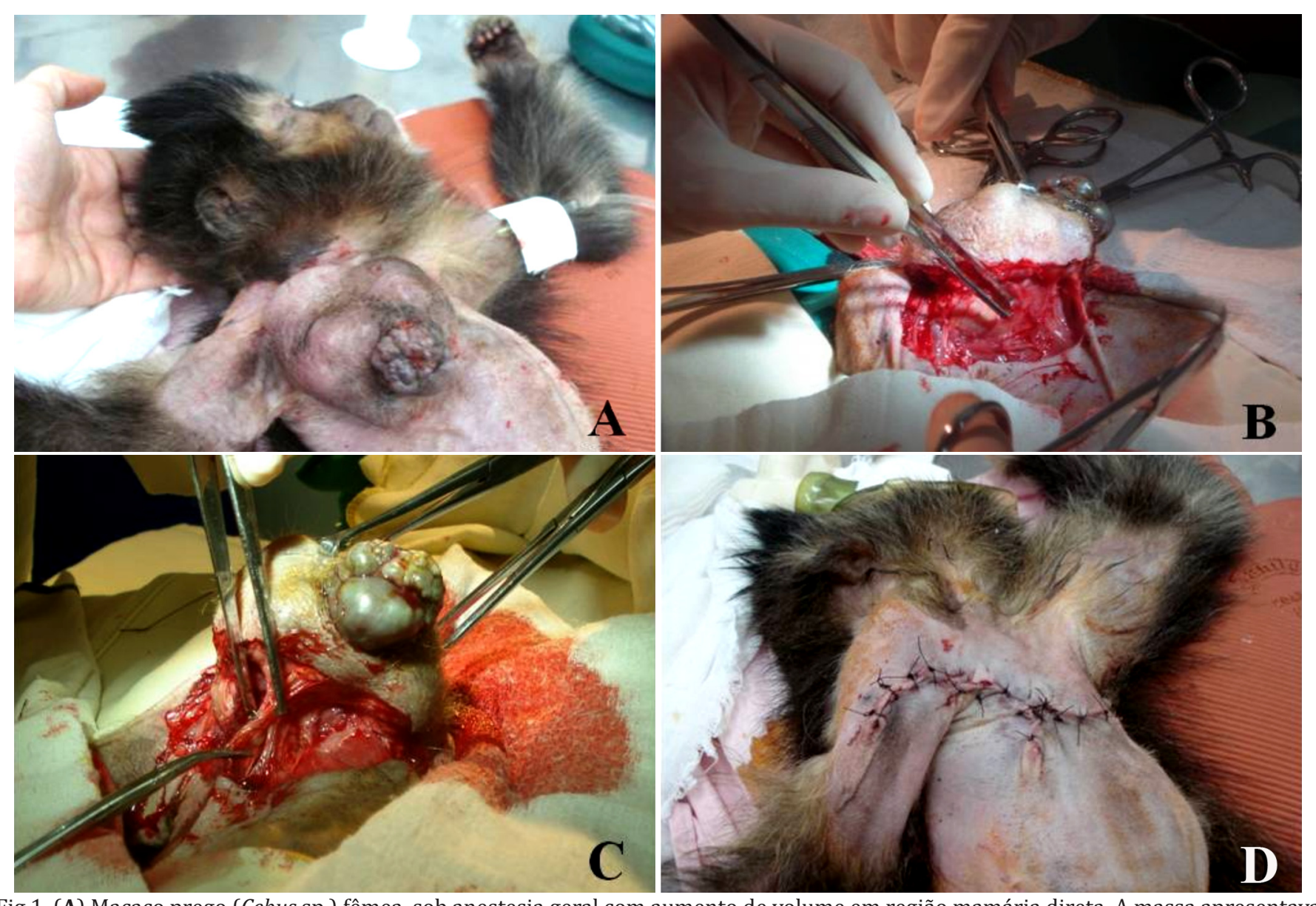

Fig.1. (A) Macaco prego (Cebus sp.) fêmea, sob anestesia geral com aumento de volume em região mamária direta. A massa apresentava $7 \mathrm{~cm}$ de altura por $7,5 \mathrm{~cm}$ de largura, com as características de consistência firme, aspecto irregular, indolor à palpação, aderida à musculatura, com alteração da morfologia do mamilo que apresentava pequenas ulcerações e extensão para região axilar direita. Imagem ilustra paciente em decúbito dorsal e membro torácico estendido lateralmente no momento da preparação para o procedimento cirúrgico. (B) Dissecção de massa tumoral com a divulsão de pele e subcutâneo ao redor de tecido mamário anômalo. (C) Secção de músculo peitoral superficial com hemostasia de pequenos vasos. (D) Aspecto pós-operatório imediato. 


\section{RELATO DE CASO}

O Serviço de Atendimento a Animais Selvagens (SAAS) é um dos serviços que formam a Clínica Escola Veterinária (Cevet) da Universidade Estadual do Centro-Oeste, localizada na cidade de Guarapuava, estado do Paraná. Todos os animais que são encaminhados ao SAAS passam por um período de quarentena. No caso de primatas o período de observação serve principalmente para avaliação de sinais clínicos referentes à Febre Amarela que é endêmica no interior do Paraná. Uma fêmea adulta de macaco-prego (Cebus sp.) foi recebida pelo Serviço de Atendimento de Animais Selvagens (SAAS) encaminhado pela Polícia Florestal (Força Verde). 0 animal era mantido em cativeiro ilegal, sendo apreendido pelo Instituto Ambiental e Polícia Ambiental na cidade de Pitanga, interior do Paraná. À inspeção inicial observou-se o aumento de volume em região mamária direita e, após contenção físico-química (Física: puçá e luva de couro. Química: cetamina na dose de $7 \mathrm{mg} / \mathrm{kg}$ e midazolan na dose de $0,5 \mathrm{mg} / \mathrm{kg}$, ambos pela via intramuscular) realizou-se o exame clínico completo e os exames complementares, determinando-se as suspeitas diagnósticas e possíveis terapias a serem empregadas. Por fim, o tratamento cirúrgico foi escolhido. Ao exame clínico foi evidenciado o aumento de volume na glândula mamaria direita com $7 \mathrm{~cm}$ de altura por $7,5 \mathrm{~cm}$ de largura, de consistência firme, aspecto irregular, indolor à palpação, aderida à musculatura, com alteração da morfologia do mamilo que apresentava pequenas ulcerações e extensão para região axilar direita (Fig.1A). Nenhuma outra anormalidade foi observada no exame clinico. Ao exame radiográfico (Fig.2) foi observada a presença de três pontos de radiopacidade, característicos de projétil balístico de arma de pressão ("chumbinho"). Um dos projéteis encontrava-se alojado ao centro da massa de tecido mamário, o que levou a suspeita de que o aumento de volume fosse consequência da reação

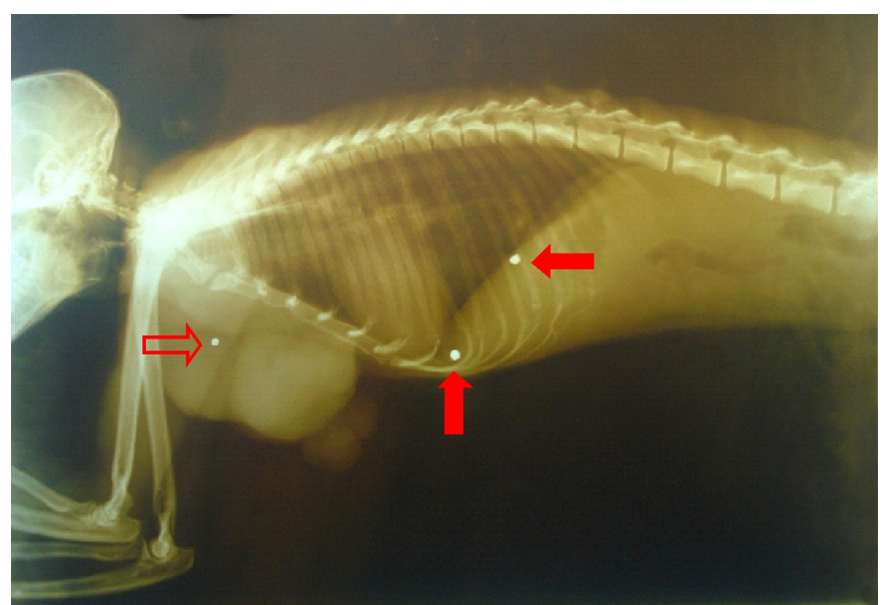

Fig.2. Imagem radiográfica em incidência látero-lateral da paciente da espécie Cebus sp. (Macaco-prego) onde se observa aumento de volume em região torácica direita (mama direita) e a presença de três nódulos semelhantes a projéteis balísticos de arma de pressão. Um dos nódulos encontra-se ao centro de massa de mama (seta vazia) e os outros dois em lateral de tórax (setas cheias). Não se observam alterações em parênquima pulmonar, não se evidenciando a presença de metástases.

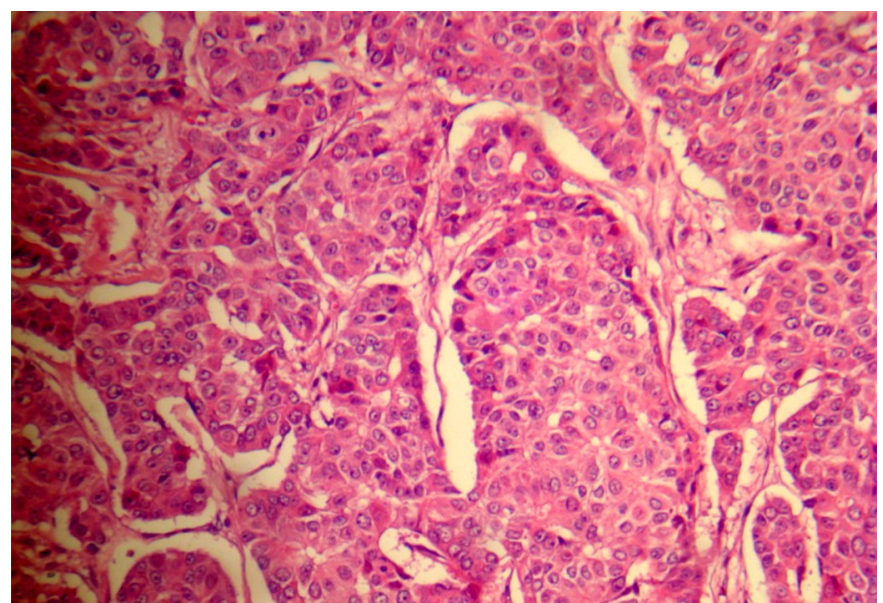

Fig.3. Aspecto histológico de carcinoma adenoescamoso em Macaco prego (Cebus sp.). Em imagem de corte histológico observam-se agrupamentos de células com núcleos amplos, ovalados, citoplasma de bordos distintos e estroma fibrovascular. HE, obj.20x

tecidual ao projétil. Além da hipótese diagnóstica de lesão inflamatória, outra possibilidade diagnóstica foi de neoplasia mamária. Após estabilização, a paciente foi submetida à exérese cirúrgica de aumento de volume, sob anestesia geral inalatória com isoflurano. 0 procedimento cirúrgico foi efetivado com o animal em decúbito dorsal e membro torácico direito estendido lateralmente (Fig.1A). Realizada a incisão elíptica ou fusiforme (tipo W. Meyer) ao redor de toda mama com margens de seguranças de aproximadamente um centímetro. A dissecção da massa foi realizada de forma semelhante à técnica de Patey (Gomes 1997, Guimarães 2007) para mastectomia em mulheres, com a dissecção da glândula mamária e secção do músculo peitoral superficial, preservando-se o músculo peitoral profundo. Após a remoção da massa com estrutura glandular e músculo peitoral, foi realizada a aproximação de musculatura, redução de espaço morto, e sutura de subcutâneo com fio poliglactina 910 (3.0) e por fim a dermatorrafia com fio de poliamida 3.0 (Fig.1B a 1D). No pós-operatório imediato foram utilizados analgésicos opioides (tramadol na dose de $1 \mathrm{mg} /$ $\mathrm{kg}$ ), antinflamatório não esteroidal (meloxican na dose de $0,1 \mathrm{mg} / \mathrm{kg}$ ) e a profilaxia antibiótica com amoxicilina associada a clavulanato $(11 \mathrm{mg} / \mathrm{kg})$. Foi realizada bandagem com esparadrapo hipoalergênico poroso fixado a pele com cola de cianoacrilato e a ferida foi protegida com "camiseta" de tecido de algodão. Os fármacos antimicrobianos (cefalexina na dose de $20 \mathrm{mg} / \mathrm{kg}$, BID) e analgésicos (meloxican na dose de $0,1 \mathrm{mg} / \mathrm{kg}$, SID) administrados no pós-operatório foram fornecidos por via oral nos alimentos (frutas e ovos cozidos). A paciente apresentou boa recuperação pós-operatória, se alimentando adequadamente, permitindo o manejo e curativo da ferida. A massa retirada foi conservada em solução de formalina a $10 \%$ e encaminhado para exame histopatológico. Ao exame histopatológico (realizado de acordo com a classificação veterinária) foi observada a presença de neoplasia maligna, invadindo a derme profunda e atingindo a camada muscular, caracterizada por cordões e agrupados de células com núcleos amplos e ovalados, nuc- 
léolos exuberantes e citoplasma de bordos indistintos. Ainda identificada a presença de numerosas figuras de mitose, focos de diferenciação escamosa, com a formação de pérolas córneas e estroma fibrovascular. 0 diagnóstico foi de carcinoma pouco diferenciado compatível com carcinoma adenoescamoso (Fig.3). Com dez dias de pós-operatório foi observada a boa cicatrização da ferida e realizada a retirada dos pontos. Após a sua completa recuperação (aproximadamente 60 dias), o animal foi encaminhado para o Centro de Triagem de Animais Selvagens (Cetas) da Pontifícia Universidade Católica do Paraná (PUC) em Tijucas do Sul, PR. A paciente foi mantida em recinto com outros animais e até a presente data (22 meses após a cirurgia) apresenta-se sem sinais de recidiva, alimentando-se bem e convivendo normalmente com o grupo observados à inspeção visual e exame físico.

\section{DISCUSSÃo}

Há inúmeros relatos de neoplasias em primatas não humanos sejam de vida livre ou de cativeiro. A maioria deles nos primatas do velho mundo e em achados de necropsia de indivíduos pertencentes a entidades responsáveis por animais em cativeiro. Segundo informações de literatura os macacos rhesus e babuínos são mais frequentemente acometidos. Nestes grupos já foram relatados tumores dos sistemas: respiratório, digestório, reprodutor, nervoso e tegumentar (Lewis \& Colgin 2005).

Nos primatas não humanos do novo mundo pode se encontrar relatos de: tumores nasais em sagui (Callithrix jacchus); tumor neuroendócrino de pulmão em macaco aranha; tumores de cavidade oral (odontoma, carcinoma de células escamosas e carcinossarcoma) em macaco-de-cheiro (Saimiri sciureus) e Cebus sp.; colangiocarcinoma em Cebus sp., tumores intestinais (adenocarcinoma de intestino delgado e de cólon e tumor estromal) em sagui, tamarin cottontop (Edipo saguinus) e macaco-aranha (Ateles sp.); nefroblastoma em sagui; feocromocitoma em tamarin (Saguinus sp.), macaco aranha e bugio (Alouatta caraya); tumores ovarianos e uterinos (leiomioma, fibromioma e teratoma) em macaco de cheiro e sagüi; tumores testiculares (células intersticiais, seminoma, de células de Sertoli e adenocarcinoma) em macaco de cheiro, macaco coruja (Aotus trivirgata) e tamarin; tumores prostáticos em macaco de cheiro, oligodendroglioma em macaco coruja; neoplasias de pele e subcutâneo (hemangioma, papiloma, lipoma, adenocarcinoma, leiomiossarcoma, carcinoma de células escamosas) em macaco-aranha, macaco-prego (Cebus apella), macaco-de-cheiro, sagui, Cebus sp. e tamarin; e doenças mieloproliferativas (leucemia e linfoma) em macaco prego e tamarin (Brown et al. 1980, Bennett et al. 1981, Grana et al. 1992, Klinger et al 1993, Hofmann et al. 2001, Haworth et al. 2003, Moutsopoulos et al. 2004, Lewis \& Colgin 2005, Cho et al. 2007, Zöller et al. 2008, Bommineni et al. 2009, Juan-Sallés et al. 2009, Miller et al. 2010, Tobi et al. 2010).

No que se refere aos tumores de mama, o maior número de relatos se refere aos primatas não humanos do velho mundo. Estas neoplasias são bem mais frequentes em humanos e foram encontrados apenas 35 relatos em primatas não humanos. Nestes relatos os tipos histológicos en- contrados foram os seguintes: adenocarcinoma, sarcoma fusocelular, tumor misto, carcinoma de células escamosas, carcinoma ductal, fibroadenoma, e fibrossarcoma (Tekeli \& Ford 1980, Hubbard et al. 1984, Waggie et al. 2000, Lewis \& Colgin 2005, Smith et al. 2005).

Esta é a primeira descrição de um caso de neoplasia mamária em Cebus sp. Há apenas um relato semelhante em primatas do novo mundo, sobre a remoção cirúrgica de uma neoplasia maligna (adenocarcinoma) em macaco de cheiro, descrito por Waggie et al. (2000). Estes autores afirmam ainda, que a paciente obteve boa sobrevida após o tratamento. Pela baixa incidência de neoplasias mamárias relatadas em primatas não humanos sugere-se que ela pode não sofrer, em sua indução, influência hormonal ou estímulo estrogênico de forma relevante como é observado em cadelas, gatas e mulheres (Gomes 1997, Lana et al. 2007). Ainda, sabe-se que materiais estranhos como implantes metálicos, de polímero, cerâmica e de silicone podem induzir o câncer e são implicados na carcinogênese em medicina humana e em medicina veterinária (McGregor et al. 2000, Henry 2007) e, desta forma, acredita-se que a presença de corpo estranho (projétil balístico) na glândula mamária possa ter influenciado na indução da neoplasia na paciente atendida.

Apesar da alta incidência de tumores de mama em mulheres, o tipo histológico de carcinoma adenoescamoso é raro. O carcinoma adenoescamoso de mama em geral é caracterizado por ser bem circunscrito, integrante do grupo dos carcinomas metaplásicos, associados à mutação do antioncogene BRCA1 e ao bom prognóstico (Ho et al 2006, Geyer et al. 2010, Noël et al. 2010). Independente do tipo histológico, tanto em mulheres como em outros animais como cadelas, gatas e possivelmente em primatas não humanos, o tratamento para as neoplasias malignas de mama deve ser realizado de forma precoce para a obtenção de melhor prognóstico (Gomes 1997, Lana et al. 2007, Morris \& Dobson 2007). De acordo com o sistema de classificação dos tumores malignos do Ministério da Saúde/INCA, as neoplasias malignas devem ser classificadas de acordo com a extensão do tumor primário (T), a ausência ou presença de linfonodos regionais metastáticos $(\mathrm{N})$ e à ausência ou presença de metástases à distância (M). Esta classificação chamada de TNM tem por finalidade o estadiamento clínico dos pacientes com câncer e auxiliar no planejamento do tratamento, dar indicações de prognóstico, ajudar a avaliação de resultados, facilitar a troca de informações e contribuir para a pesquisa (Brasil 2004). Este tipo de classificação também é utilizado para animais domésticos como cães e gatos e, de acordo com a classificação TNM, o tamanho do nódulo tumoral apresenta grande importância na decisão da terapia apropriada e prognóstico dos pacientes, assim como o tipo histológico da neoplasia (Lana et al. 2007, Morris \& Dobson 2007).

Em primatas não humanos não há dados correlacionando as características do tumor e tipo histológico com tempo de sobrevida e agressividade. Contudo, mesmo não havendo dados para a correlação entre classificação da TNM e terapia adequada, neste caso, foi optado pela cirurgia semelhante à técnica adotada em seres humanos com a exé- 
rese da massa tumoral com margens de segurança. Como as estruturas anatômicas da glândula mamária dos primatas humanos e não humanos são semelhantes entre si foi optado pela adoção da técnica cirúrgica semelhante à realizada em mulheres e não a semelhante a cadelas ou gatas. Desta forma foi realizada a remoção da glândula mamária com o músculo peitoral superficial. Não foi realizado o esvaziamento axilar (linfonodectomia de cadeia axilar) já que em inspeção de tecido glandular e linfático de região não se observou aumento de volume ou alterações macroscópicas. Ainda, no momento da cirurgia não havia a confirmação das características histológicas da massa e a decisão de não se realizar a exérese de linfonodos foi feita pensando-se na qualidade de vida da paciente e objetivando-se sua reintrodução em ambiente natural ou de cativeiro, preservando-se a movimentação do membro.

0 tecido excisado foi encaminhado para exame histopatológico confirmando a suspeita de neoplasia mamária maligna. Foi cogitada a possibilidade de realização de terapia adjuvante antineoplásica, porém a existência de dados bibliográficos com doses e drogas adequadas e efetivas para primatas é escassa (Hahn 2005). A ausência de dados de referência, a boa recuperação da paciente e o desejo de reintroduzi-la em ambiente natural desencorajaram a adoção de terapia antineoplásica no pós-operatório.

Mais de vinte meses após a terapia cirúrgica, o animal encontra-se em bom estado geral sugerindo que a terapia adotada foi eficiente até o momento em alcançar qualidade de vida e aumento de sobrevida.

\section{CONCLUSÃO}

A neoplasia mamária em primatas não humanos é rara e, foi encontrada apenas uma descrição de neoplasia mamária em primatas do novo mundo. Neste relato foi possível diagnosticar e tratar uma fêmea de Cebus sp. com carcinoma mamário de alto grau (sugestivo de carcinoma adenoescamoso). Os procedimentos adotados garantiram até o momento qualidade de vida e boa sobrevida.

\section{REFERÊNCIAS}

Brasil 2012. Estimativa 2012: incidência de câncer no Brasil. Ministério da Saúde, INCA, Rio de Janeiro.

Brasil 2004. TNM: Classificação de Tumores Malignos. 6ª ed. Ministério da Saúde, INCA, Rio de Janeiro.

Bicca-Marques J.C., Silva V.M. \& Gomes D.F. 2006. Família Cebidae, p.102118. In: Reis N.R., Peracchi A.L., Pedro W.A. \& Lima I.P. (Eds), Mamíferos do Brasil. Editora da Universidade Estadual de Londrina, Londrina, PR.

Bennett B.T., Beluhan F.Z. \& Sarpel S.C. 1981. Acute myelomonocytic leukemia in a Capuchin monkey (Cebus apella). Lab. Anim. Sci. 31:519-21. (Resumo)

Bommineni Y.R., Dick E.J. Jr \& Hubbard G.B. 2009. Gastrointestinal stromal tumors in a baboon, a spider monkey, and a chimpanzee and a review of the literature. J. Med. Primatol. 38(3):199-203.

Brown R.J., O’Neill T.P., Kessler M.J. \& Andress D. 1980. Cholangiocarcinoma in a Capuchin monkey (Cebus albifrons). Vet. Pathol. 17(5):626-629.

Cho H.S., Kim Y.S., Choi C., Lee J.H., Kurkure N.V., Subramanian M. \& Park N.Y. 2007. Neuroendocrine tumor in the lung of a captive black spider monkey (Ateles paniscus). J. Vet. Med. Sci. 69(7):783-5.

Geyer F.C., Lambros M.B., Natrajan R., Metha R., Mackay A., Savage K., Parry S., Ashworth A., Badve S. \& Reis-Filho J.S. 2010. Genomic and immuno- histochemical analysis of adenosquamous carcinoma of the breast. Mod. Pathol. 23(7):951-960.

Gomes R. 1997. Câncer de mama, p.184-196. In: Gomes R. (Ed.), Oncologia Básica. Editora Reninter, Rio de Janeiro.

Guimarães J.S. 2007. Cirurgia das glândulas mamárias, p.314-325. In: Goffi F.S. (Ed.), Técnica Cirúrgica: bases anatômicas, fisiopatológicas e técnica cirúrgica. $4^{a}$ ed. Atheneu, São Paulo.

Grana D., Mareso E. \& Gomez E. 1992. Oral squamous cell carcinoma in capuchin monkeys (Cebus apella): Report of two cases. J. Med. Primatol. 21(7/8):384-386. (Resumo)

Hahn K.A. 2005. Chemotherapy dose calculation and administration in exotic animal species. Semin. Avian Exotic Pet Med. 14:193-198.

Haworth R., Jones S., Sanchez-Morgado J. \& Pilling A. 2003. Ovarian teratoma in a common marmoset (Callithrix jacchus). Vet. Rec. 153(11):332333. (Resumo)

Henry C.J. 2007. The etiology of cancer, p.12-19. In: Withrow S.J. \& Vail D.M. (Eds), Small Animal Clinical Oncology. $4^{\text {th }}$ ed. W.B. Saunders, St Louis.

Ho B.C., Tan H.W., Lee V.K. \& Tan P.H. 2006. Preoperative and intraoperative diagnosis of low-grade adenosquamous carcinoma of the breast: Potential diagnostic pitfalls. Histopathol. 49(6):603-611.

Hofmann P., Kahnt K., Mätz-Rensing K., Brack M. \& Kaup F.J. 2001. Three spontaneous lymphomas in a colony of cotton-top tamarins (Saguinus oedipus). J. Med. Primatol. 30(6):322-327.

Hubbard G.B., Wood D.H. \& Butcher W.I. 1984. Mammary carcinoma with metastasis in a rhesus monkey (Macaca mulatta). Vet. Pathol. 21:531533.

Juan-Sallés C., Ramos-Vara J.A. \& Garner M.M. 2009. Pheochromocytoma in six New World primates. Vet. Pathol. 46(4):662-666.

Klinger M.M., Levee E.M. \& Scholes J.V. 1993. Giant fatty tumor in a Cebus apella. J. Med. Primatol. 22(7/8):435-436.

Koehler A. \& Firkowski C. 1996. Descascamento de pinus por macaco-prego (Cebus apella). Floresta. 24:61-75.

Lana S.E., Rutteman G.R. \& Withrow S.J. 2007. Tumors in the mammary gland, p.619-636. In: Withrow S.J. \& Vail D.M. (Eds), Small Animal Clinical Oncology. $4^{\text {th }}$ ed. W.B. Saunders, St Louis.

Lewis A.D. \& Colgin L.M.A. 2005. Pathology of non infectious diseases of the laboratory primate, p.47-74. In: Wolfe-Cotte S. (Ed.), The Laboratory Primate. Elsevier, London.

McGregor D.B., Baan R.A., Partensky C., Rice J.M. \& Wilbourn J.D. 2000. Evaluation of the carcinogenic risks to humans associated with surgical implants and other foreign bodies: A report of an IARC Monographs Program Meeting, International Agency for Research on Cancer. Eur. J. Cancer 36:307-313.

Morris J.M. \& Dobson J. 2007. Glândula mamária, p.185-192. Morris J.M. \& Dobson J. (Eds), Oncologia em Pequenos Animais. Roca, São Paulo.

Miller A.D., Kramer J.A., Lin K.C., Knight H., Martinot A. \& Mansfield K.G. 2010. Small intestinal adenocarcinoma in common marmosets (Callithrix jacchus). Vet. Patho. 47:969-976.

Moutsopoulos N.M., Nikitakis N.G., Powell D.A. \& Reynolds M.A. 2004. Carcinosarcoma of the maxilla in a squirrel monkey (Saimiri sciureus). Comp. Med. 54(3):333-336.

Noël J.C., Bruxant F. \& Engohan-Aloghe C. 2010. Low-grade adenosquamous carcinoma of the breast: A case report with a BRCA1 germline mutation. Pathol. Res. Pract. 206(7):511-513.

Passos F.C., Miranda J.M.D., Aguiar L.M., Ludwig G., Bernardi I.P. \& Moro-Rios R.F. 2006. Distribuição e ocorrência de primatas no estado do Paraná, Brasil, p.119-149. In: Bicca-Marques J.C. (Ed.), A Primatologia no Brasil. Vol.10. Edipucrs, Porto Alegre. (Lançado na Conferência da Sociedade Brasileira de Primatologia)

Rocha V.J., Reis N.R. \& Sekiama M.L. 1998. Uso de ferramentas por Cebus apella Linnaeus (Primates, Cebidae) para a obtenção de larvas de Coleóptera que parasitam sementes de Syagrus romanzoffianum (Cam.) Glassm. (Arecaceae). Revta Bras. Zoologia 15(4):945-950.

Rocha V.J. 2000. Macaco-prego, como controlar esta nova praga florestal? Floresta 30: 95-99. 
Smith J.M., Rao S.S., Stump K.C., Benazzi C., Sarli G. \& DeTolla L.J. 2005. Mammary ductal carcinoma with comedo pattern in a rhesus macaque. Comtemp. Top. Lab. Anim. Sci. 44:29-33.

Tekeli S. \& Ford T.M. 1980. Spontaneous intraductal mammary carcinoma in a rhesus monkey. Vet. Pathol. 17:502-504.

Tobi M., Kim M., Zimmer R., Hatfield J., Kam M., Khoury N., Carville A., Lawson M.J., Schiemann W.P. \& Thomas P. 2010. Colorectal cancer in the cotton top tamarin (Saguinus oedipus): How do they evade liver metastasis? Dig. Dis. Sci. 56:397-405.
Verona C.E.S. \& Pissinatti A. 2006. Primatas do novo mundo, p.358-377. In: Cubas Z.S., Silva J.C.R. \& Catão-Dias J.L. (Eds), Tratado de Animais Selvagens. 1 a ed. Roca, São Paulo.

Waggie K.S., Tolwani R.J. \& Lyons D.M. 2000. Mammary adenocarcinoma in a male squirrel monkey (Saimiri sciureus). Vet. Pathol. 37:505507.

Zöller M., Mätz-Rensing K., Fahrion A. \& Kaup F.J. 2008. Malignant nephroblastoma in a common marmoset (Callithrix jacchus). Vet. Pathol. 45(1):80-84. 